\title{
Comparing Methods for Developing Site Index Prediction Model of Sugi (Cryptomeria japonica) Planted Stand
}

\author{
Yasushi Mitsuda ${ }^{1 *}$, Hiroki Tsutsumi ${ }^{1}$
}

\begin{abstract}
This study aimed to compare three methods for developing site index prediction model for specific species for a given site using environmental factors as explanatory variables. The following methods were compared in this study: (1) guide curve based method, (2) difference equation based method, and (3) combined site index prediction and height growth curve models method. The site index models were parameterized by the three methods with a permanent plot data and then evaluated for accuracy and practicability in model prediction using the stem analysis data. Root mean squared error and Spearman's rank correlation coefficient were used as statistics representing model accuracy and practicability; values obtained for the model derived from combined method were 4.468 and 0.754 , respectively, which indicated that it was the best among the three models.
\end{abstract}

Keywords: Bayesian calibration, Cryptomeria japonica, height growth curve, site index

\section{Introduction}

Site productivity maps for merchantable species are essential tools for landscape-scale forestry (Kayahara et al., 1998; Klinka and Feller, 1984; Mitsuda et al., 2003; Takeshita et al., 1966). For example, a site productivity map for sugi (Cryptomeria japonica) was used as an indicator for the suitability of timber production in forest management objective zoning, which prioritize forest management objectives (timber production, natural forest conservation and restoration, and harmonization of both) for each stand (Mitsuda et al., 2013). Re-plantation after clear-cutting has become an urgent issue in Japan, with the increase in clear-cutting, and site productivity maps are important for decisions on re-plantation. Developing a site index prediction model for mapping spatial distribution of site index is an essential issue in forestry practices.

Measuring site index, which is most widely and commonly used for productivity evaluation in forestry (e.g., Davis and Johnson, 1987; Hägglund, 1981; Monserud et al., 1990; Takeshita et al.,1960; Wang, 1995), is challenging and causes problems in developing site index prediction models (Mitsuda et al., 2007; Mitsuda, 2014). Site index is defined as the dominant tree height at a reference age (Davis and Johnson, 1987) (40 years is commonly used in Japan); field measurement of dominant tree height in stands of the reference age or stem analysis are limited ways for accurately measuring the site index (e.g., Kayahara et al., 1998; Mitsuda et al., 2001; 2007; Monserud et al.,1990; Wang, 1995). Acquiring enough data for developing site index prediction model using these two methods is possible but difficult. Therefore, estimated site index data derived from the field-measured dominant tree height in stands of any age have been used to develop site index prediction models (e.g., Auten, 1945; Chen and Abe, 1999; Curt et al., 2001; Green et al., 1989; Trimble and Weitzman, 1956).

There are several ways to estimate the site index, and which is used as the objective variable for developing site index prediction models, but the guide curve technique is the most commonly used technique using dominant tree height data from stands of various ages (Davis and Johnson, 1987). The technique is based on standard height growth curves, called guide curves, which are parameterized using the average dominant tree height of stands of each age class. When applying the guide curve technique, we need to group the individual site data into age class, which causes a loss of individual site information. The other major technique for estimating site index is the difference equation technique, which uses time series data derived from repeated measurements (Clutter et al., 1983). This technique uses individual site information; however, repeatedly measured field survey data is not ordinary data and therefore this technique have been not used in actual forestry practices. Therefore, these two techniques have problems in data processing and collection. Conventional methods for developing a site index prediction model have applied the guide curve or difference equation technique for estimating site index for each sample site, and then developed site index

Received Nov. 13, 2017; Accepted Feb. 21, 2018

${ }^{1}$ University of Miyazaki, Japan

*Corresponding Author: mitsuda@cc.miyazaki-u.ac.jp 
prediction models using estimated site index as the objective variable, therefore there are same problems which are loss of individual site information and need for repeatedly measured field survey data.

A new method for developing a site index prediction model was proposed to overcome the drawbacks of methods based on these two techniques for estimating site index for a site (Mitsuda, 2014). The method has some advantages over the existing ones, however, their performances have not been examined and compared with previous two methods using actual data. If site index prediction models developed using this new method can provide acceptable predictions, it would become an attractive alternative for developing site index prediction model. Therefore, performances of the newly proposed and two traditional methods should be evaluated and compared in terms of accuracy and practicability in site index predictions. The objectives of this study were to develop site index prediction models using these three methods, evaluate their accuracy and practicability for predictions, and discuss the advantages of the newly proposed method over the two traditional methods.

\section{Materials and Method}

\subsection{Methods for developing site index prediction model}

Three methods for developing site index prediction models were compared: (1) guide curve technique based (GC) method, (2) difference equation technique based (DE) method, and (3) combined height growth curve and site index prediction models (COM) method (Figure 1).

(1) GC method

Field measurement
(2) DE method

Field measurement: $\mathrm{t} 1$

\section{(3) COM method}

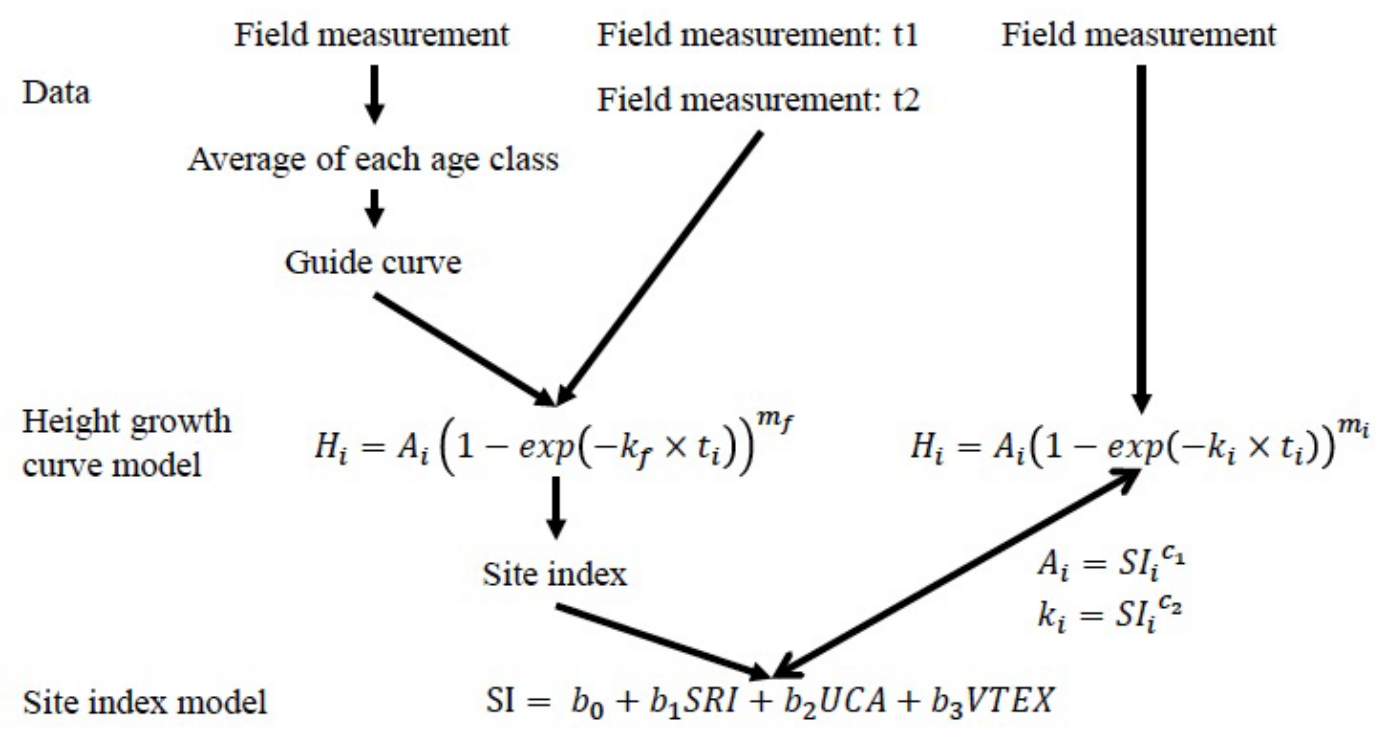

Figure 1. Comparison of the three methods for developing site index prediction models.

The three methods for developing a site index prediction model consist of two parts, which are site index estimation and site index prediction model development. Tree height growth curve models, which describe the trajectory of tree height growth, were used for estimating site index for a site in the three methods. In this study, Richard's three parameter function was adopted as the height growth curve model (Richards, 1959):

$$
H=A(1-\exp (-k \times t))^{m}
$$

where $H$ is dominant tree height $(\mathrm{m}) ; t$ is stand age; and $A, k$, and $m$ are parameters.

Several site index prediction models have been developed for different species in different regions, where environmental factors are used as explanatory variables (e.g., Corona et al., 1998; Hunter and 
Gibson, 1984; Klinka and Carter, 1990; Nishizawa et al., 1965; Takeshita et al., 1960; Wang et al., 2004). The following linear regression model using topographic factors derived from digital terrain analysis was used as site index prediction model for this study (Mitsuda and Ito, 2015).

$$
S I=b_{0}+b_{1} S R I+b_{2} U C A+b_{3} V T E X
$$

where $S I$ is site index; $S R I$ is solar radiation index; $U C A$ is hydrological upslope contributing area index; VTEX is vertical topographic exposure index; and $b_{0}, b_{1}, b_{2}$, and $b_{3}$ are coefficients of regression. SRI indicates the intensity of solar radiation that causes soil moisture deficiency. UCA reflects the tendency of soil water accumulation. VTEX is related to wind exposure that causes the risk of forced evapotranspiration. These topographic factors were selected as the best explanatory variables for sugi site index prediction model developed in Mitsuda and Ito (2015), the details of which have been explained in the previous papers (Mitsuda et al., 2007; Mitsuda and Ito, 2015).

\subsection{GC method}

The GC method is the conventional and most commonly used method for estimating site index and then developing a site index prediction model (e.g., Auten,1945; Chen and Abe, 1999; Curt et al., 2001; Green et al., 1989; Trimble and Weitzman, 1956). Parameter $A$ of the height growth curve (Eq. [1]) was treated as a site-specific parameter in relation with site productivity, and parameters $k$ and $m$ were fixed, thus the shapes of the height growth curves for various sites were similar, and only the maximum tree heights were different between sites. The height growth curve model for GC method is represented as follows (Davis and Johnson, 1987):

$$
H_{i}=A_{i}\left(1-\exp \left(-k_{f} \times t_{i}\right)\right)^{m_{f}}
$$

where subscription $i$ means the $i$ th data; and $k_{f}$ and $m_{f}$ are common parameters for all data. For estimating $k_{f}$ and $m_{f}$, field measurement data are divided into age class with 5-years intervals, and average dominant tree height and stand age of each age class were calculated, followed by applying nonlinear least squares regression to develop the standard height growth curve:

$$
\bar{H}_{j}=A_{s}\left(1-\exp \left(-k_{f} \times \bar{t}_{j}\right)\right)^{m_{f}}
$$

where $\bar{H}_{j}$ and $\bar{t}_{j}$ are average dominant tree height and average stand age of $j$ th age class, respectively, and $A_{s}$ is the parameter of the standard height growth curve (i.e., guide curve).

Using estimated parameters $k_{f}$ and $m_{f}$, parameter $A_{i}$ and then site index of each site can be estimated by the following functions:

$$
\begin{aligned}
A_{i} & =\frac{H_{i}}{\left(1-\exp \left(-k_{f} \times t_{i}\right)\right)^{m_{f}}} \\
S I_{i} & =A_{i}\left(1-\exp \left(-k_{f} \times t_{r e f}\right)\right)^{m_{f}}
\end{aligned}
$$

where $S I_{i}$ is site index of $i$ th data; and $t_{r e f}$ is the reference age.

The estimated site index data were used for estimating parameters of the prediction model (Eq. [2]) using topographic factors by linear least squares regression.

\subsection{DE method}

The height growth curve model used for the DE method was same as that for the GC method. The time series data derived from repeated field measurement were used for estimating $k_{f}$ and $m_{f}$ parameters using the fractional form of tree height growth curves (Clutter et al., 1983).

$$
\begin{aligned}
& \frac{H_{i, 2}}{H_{i, 1}}=\frac{A_{i}\left(1-\exp \left(-k_{f} \times t_{i, 2}\right)\right)^{m_{f}}}{A_{i}\left(1-\exp \left(-k_{f} \times t_{i, 1}\right)\right)^{m_{f}}} \\
& H_{i, 2}=H_{i, 1} \frac{\left(1-\exp \left(-k_{f} \times t_{i, 2}\right)\right)^{m_{f}}}{\left(1-\exp \left(-k_{f} \times t_{i, 1}\right)\right)^{m_{f}}}
\end{aligned}
$$

where $H_{i, 1}$ and $H_{i, 2}$ are dominant tree heights of the $i$ th site at first and second measurements, respectively. The parameter $A_{i}$ on the right hand side of Eq. [7] can be canceled; thus, parameters 
$k_{f}$ and $m_{f}$ in Eq. [8] can be estimated using the nonlinear least squares regression. After estimating parameters $k_{f}$ and $m_{f}$, the same procedure as the GC method was applied for estimating the site index for each site and developing the site index prediction model.

\subsection{COM method}

The COM method was newly proposed in the previous paper (Mitsuda, 2014). It simultaneously parameterizes the height growth curve and site index prediction models treating site index as a hidden variable. The height growth curve model for COM method assumes that parameters $k$ and $m$ vary across sites.

$$
H_{i}=A_{i}\left(1-\exp \left(-k_{i} \times t_{i}\right)\right)^{m_{i}}
$$

Comparing with Eq. [3] adopted in the GC and DE methods, the shapes of the height growth curves were different across sites. In the COM method, the height growth curve and site index prediction models were combined as follows:

$$
\begin{array}{ll}
{[10]} & A_{i}=S I_{i}^{c_{1}} \\
{[11]} & k_{i}=S I_{i}^{c_{2}} \\
{[12]} & m_{i}=\frac{\log \left(S I_{i} / A_{i}\right)}{\log \left(1-\exp \left(-k_{i} \times t_{r e f}\right)\right)}
\end{array}
$$

where $c_{1}$ and $c_{2}$ are parameters. Parameters $A$ and $k$ are represented as functions of the site index, treated as a hidden variable derived from the site index prediction model, and $m$ is transformed to ensure that the predicted height at the reference age will be equal to the site index. Bayesian calibration using the Markov chain Monte Carlo (MCMC) method (Van Oijen et al., 2005)was applied to parameterize $b_{0}, b_{1}, b_{2}$, and $b_{3}$ of the site index prediction model (Eq. [2]) and $c_{1}$ and $c_{2}$ of the height growth curve model (Eq. [10] and [11]). A detailed parameterization with Bayesian calibration was explained in the previous paper (Mitsuda, 2014).

\subsection{Data analysis}

The study area of this study included the Tano Forest Science Station, University of Miyazaki, Japan, and the target species was sugi (Cryptomeria japonica). The reference age was set as 40 years. The field measurement data from 29 permanent plots established in sugi planted forests were used for model parameterization (Table 1).

Table 1. Summary of permanent plots and trees used for stem analysis.

\begin{tabular}{rlrrr}
\hline & & \multicolumn{2}{c}{ Permanent plot } & Stem analysis \\
\cline { 2 - 4 } & & 2007 & 2012 & \\
\hline Stand age & [years] & & & \\
& minimum & 20.0 & 25.0 & 42.0 \\
& average & 45.1 & 50.1 & 44.9 \\
& maximum & 92.0 & 97.0 & 53.0 \\
Dominant tree height & {$[\mathrm{m}]$} & & & \\
& minimum & 13.9 & 17.2 & 14.3 \\
& average & 23.3 & 25.1 & 20.9 \\
& maximum & 31.5 & 32.7 & 25.9 \\
\hline
\end{tabular}

The permanent plots were measured in 2007 and 2012. Data for 2012 were used for the GC and COM methods regarded as a temporal plot data used in the usual procedure of forestry practices, whereas that for both 2007 and 2012 were used for the DE method, which required time series data derived from repeated measurements. The average height of the three tallest trees on each plot was defined as the dominant tree height. The relationship between dominant tree height and stand age of 29 permanent plots of sugi planted forest were measured in 2007 and 2012 is shown in Figure 2.

The stem analysis data of sugi dominant trees felled at 18 sites were used for model validation (Table 1). The details of stem analysis were explained in the previous paper Mitsuda et al.(2007). 


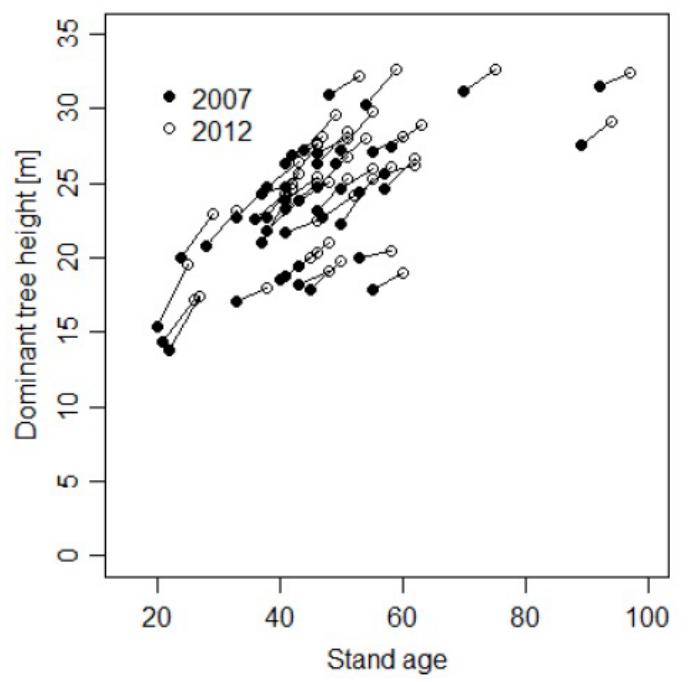

Figure 2. Relationship between dominant tree height and stand age measured in 29 permanent plots.

Tree height at 40 years observed by stem analysis for each sample tree was regarded as the observed site index value for each site. The locations of permanent plots and sites where sample trees were felled for stem analysis were recorded using DGPS (Trimble, Pathfinder ProXH), and topographic factors of each site were calculated using the 50 and 10-m resolution digital terrain model (DEM) published by the Japanese Geographic Survey Institute.

Root mean squared error (RMSE) and Spearman's rank correlation coefficient $(\rho)$ were used as statistics to evaluate the accuracy and practicability of model prediction derived from the three methods. RMSE has been used as an indicator for representing the accuracy of model prediction. Because relative evaluation of site productivity is important in forest management, $r$ and $\rho$ were used as indices of practicability. For evaluating model development using field-measured permanent plot data, the site index for each permanent plot was predicted using the site index prediction model with estimated parameters, and then the dominant tree height in 2012 for each permanent plot was predicted using height growth curve model with estimated parameters. In GC and DE methods, $A_{i}$ was estimated using Eq. [6] with parameters $k_{f}$ and $m_{f}$, and predicted site index for each plot $\left(S I_{i}\right)$ using Eq. [2]; then, dominant tree height for each plot $\left(H_{i}\right)$ was estimated using Eq. [5].

In the COM method, parameters $k_{i}, m_{i}$, and $A_{i}$ were calculated using Eqs. [10], [11], and [12] with parameters $c_{1}$ and $c_{2}$ and predicted site index for each $\operatorname{plot}\left(S I_{i}\right)$ by Eq. [2]. Model accuracy and practicability were calculated for each method using the observed and predicted dominant tree height of each plot. Because exact site index was observed for each site using stem analysis, site index prediction models derived from the three methods were directly examined. For model validation with stem analysis data, the site index for each stem analysis site was predicted using the site index prediction model (Eq. [2]) with estimated parameters for each method, and model accuracy and practicability were calculated using observed and predicted site indices for each site.

\section{Results}

In the GC method, parameters $k_{f}$ and $m_{f}$ were estimated using nonlinear least squares regression by applying Eq. [4] to the data set for average dominant height of each age class (Table 2).

Figure 3 shows the estimated guide curve. In the DE method, parameters $k_{f}$ and $m_{f}$ were estimated using nonlinear least squares regression by applying Eq. [8] to the data set of repeatedly measured dominant tree heights and stand ages for each permanent plot (Table 2). Parameters $b_{0}$, $b_{1}, b_{2}$, and $b_{3}$ were parameterized using the estimated site index and topographic factors for each permanent plot with linear regression in the GC and DE methods (Table 3 ).

In the COM method, parameters for site index prediction $\left(b_{0}, b_{1}, b_{2}\right.$, and $\left.b_{3}\right)$ and height growth curve $\left(c_{1}\right.$ and $\left.c_{2}\right)$ models were directly estimated using the Bayesian calibration using MCMC (Tables 
Table 2. Parameters of height growth curve models developed using the three methods.

\begin{tabular}{lrr}
\hline Method & $k$ & $m$ \\
\hline GC & 0.021 & 0.735 \\
DE & 0.014 & 0.946 \\
& $\mathrm{c}_{1}$ & $\mathrm{c}_{2}$ \\
COM $^{*}$ & 1.132 & -1.184 \\
\hline
\end{tabular}

* Values were medians of MCMC samples.

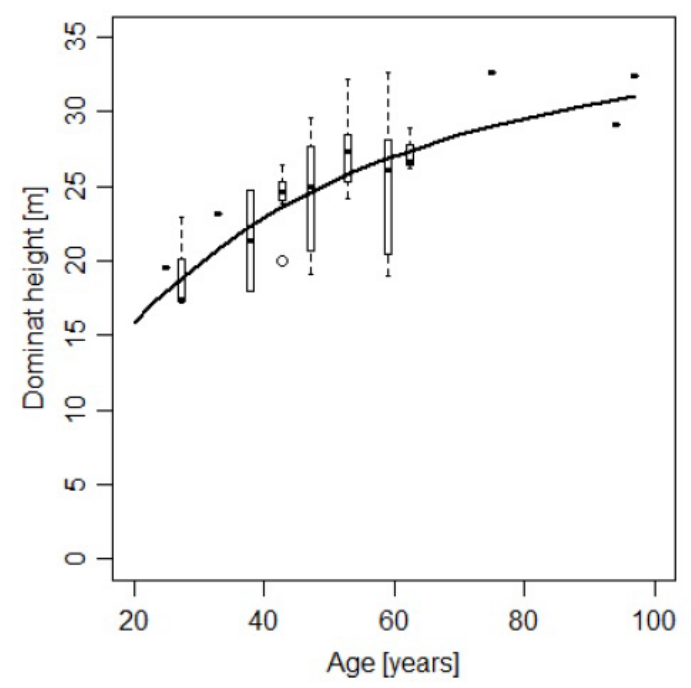

Figure 3. Guide curve developed using permanent plot data.

$2,3)$, and diagnostic statistics $(\hat{R})$ of all target parameters were below 1.1, which suggested that all parameters were converged (Gelman et al., 2003).

RMSE and $\rho$ were calculated for each model derived from each method using observed and predicted dominant tree heights in 2012 for each plot to evaluate the fitness of these models for model development (Table 4).

Figure 4 show the relationship between observed dominant tree heights for permanent plots in 2012, which were used as the objective variable of parameterization, and predicted dominant tree heights for each method. Values of RMSE were not different among all models and explicit bias was not observed.

RMSE and $\rho$ were calculated for each model derived from each method using observed and predicted site index for each plot to evaluate robustness in ranking relative productivity among sites of models to data for model validation (Table 4). Figure 5 show the relationship between observed site index of sample site of stem analysis, which was used as model validation data, and predicted site index for each method. Values of RMSE were 5.394, 4.656, and 4.468 for GC, DE, and COM methods, respectively. There was explicit over estimation for all models. The indices of practicability were the highest in the COM method $(r=0.858$ and $\rho=0.754)$.

Table 3. Parameters of site index prediction models developed using the three methods.

\begin{tabular}{lrrrrr}
\hline Method & $b_{0}$ & $b_{1}$ & $b_{2}$ & $b_{3}$ & $R^{2}$ \\
\hline GC & 19.55 & -0.337 & 1.486 & -4.653 & 0.388 \\
DE & 13.57 & 0.388 & 1.248 & -4.570 & 0.306 \\
COM $^{*}$ & 21.79 & -0.561 & 1.164 & -2.708 & \\
\hline
\end{tabular}

* Values were medians of MCMC samples. 

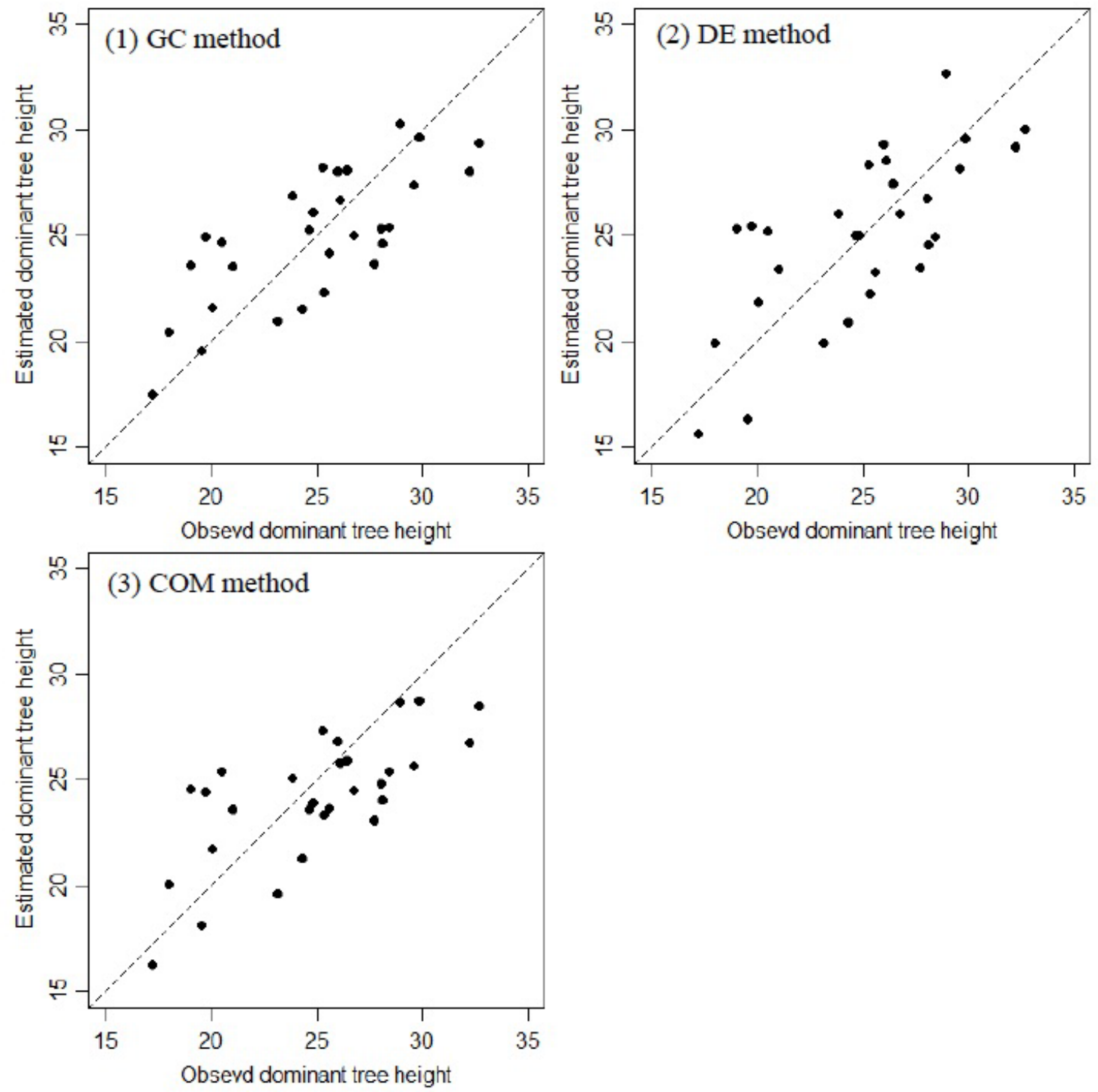

Figure 4. Relationship between observed dominant tree heights for permanent plots at in 2012. 

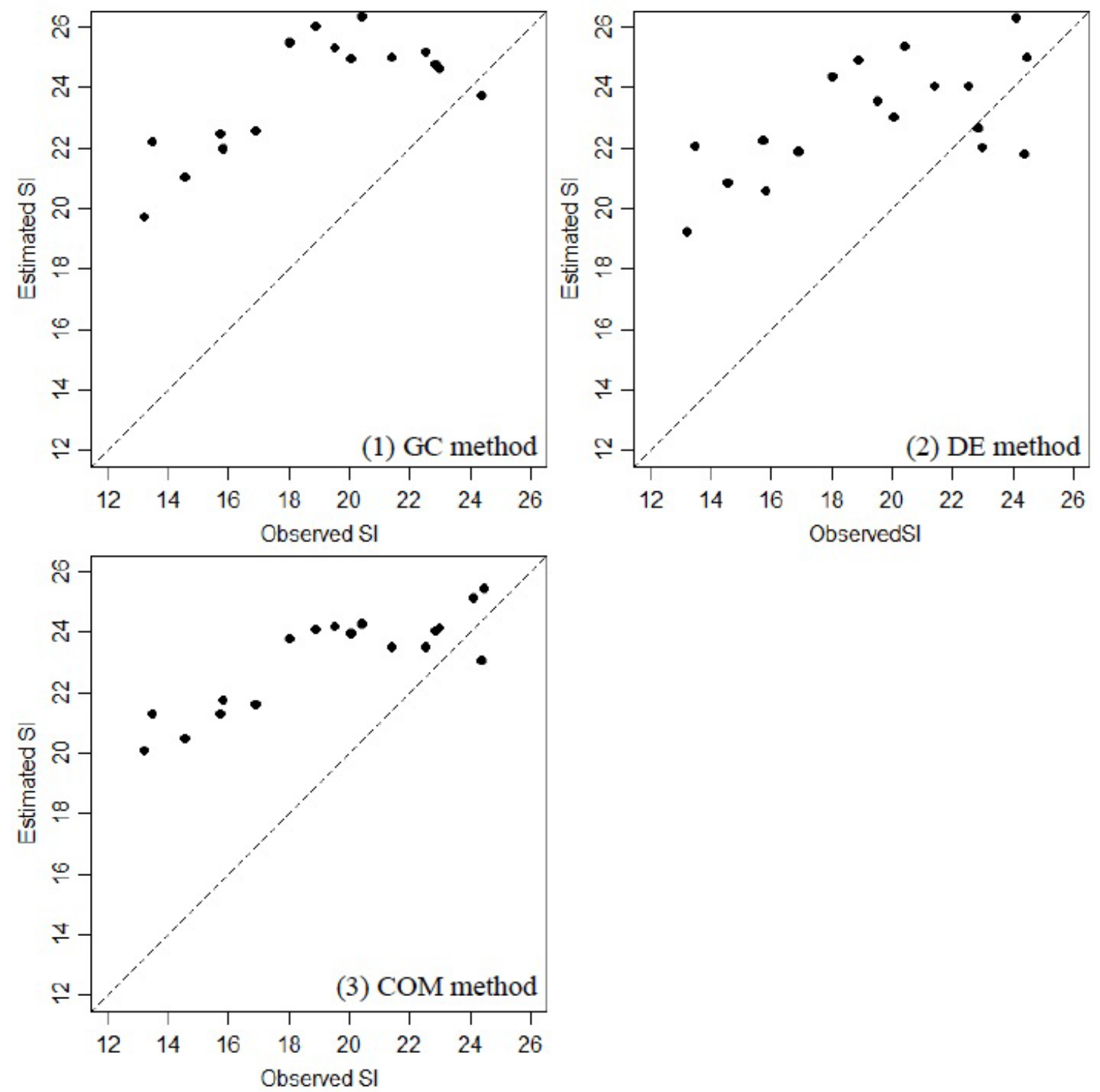

Figure 5. Relationship between observed dominant tree heights for sample site of stem analysis. 
Table 4. Statistics for evaluating accuracyand practicability of model prediction.

\begin{tabular}{lrrrrr}
\hline & \multicolumn{2}{c}{ Development } & & \multicolumn{2}{c}{ Validation } \\
\cline { 2 - 3 } \cline { 5 - 6 } Method & RMSE & $\rho$ & & RMSE & $\rho$ \\
\hline GC & 2.726 & 0.733 & & 5.394 & 0.659 \\
DE & 3.039 & 0.680 & & 4.656 & 0.512 \\
COM $^{*}$ & 2.988 & 0.685 & & 4.468 & 0.754 \\
\hline
\end{tabular}

* All statistics were calculated using medians of MCMC samples.

\section{Discussion}

Comparing the GC and DE methods, the COM method could provide an equally reliable site index prediction model. Focusing on model validation, RMSE was the lowest and $\rho$ were the highest for the COM-derived model. This suggests that models developed by the COM-derived model were robust in evaluating relative site productivity, and predicted values using these models were equally reliable compared with models derived by the two traditional methods.

Explicit over-estimation was observed when three developed models were applied to site index data derived from stem analysis for model validation. This estimation bias may have resulted due to bias in the location of the permanent plots. Observed site index by stem analysis ranged from 13.18 to 24.46 , with an average of 19.40 , while estimated site index ranged from 16.07 to 28.57 with an average of 23.19 using the GC method, from 14.44 to 29.40 with an average of 22.19 using the DE method, and from 18.34 to 25.79 with an average of 22.91 using the COM method. All models had higher estimated site index values for permanent plots, suggesting that permanent plots tended to be established in good sites. Site index prediction models developed using data from sites with higher site indices will generally predict higher site index values.

This study showed that performance of the site index model developed using the COM method was equal or superior to those of models developed using the GC and DE methods. Therefore, the COM method can be used as an alternative for developing site index prediction models. The GC method loses individual site information and uses average dominant height of each age class in developing height growth curve models, which can be a risk for developing excessively smoothed height growth curves.

Because the time series data derived from repeated measurements on permanent plots is limited, the application of the DE method is limited. The COM method thus was proposed to overcome the drawbacks of the GC and DE methods. The COM method can utilize individual site information to develop height growth curve models and can be applied to ordinal field measurement data (Figure 1). Furthermore, height growth curve models derived from the GC and DE methods assume that shapes of height growth curves do not vary with site index (i.e., monomorphic curve), whereas the COM method adopts the polymorphic height growth curve in which shapes of height growth curves vary with site index. Past studies have shown the advantages of polymorphic height growth curves over monomorphic curves (Kayahara et al., 1998; Mitsuda and Kitahara, 2015). However, the greatest barrier in utilizing the COM method is the application of the Bayesian calibration using the MCMC method. Recently, Bayesian calibration using the MCMC method became easy to implement with the development of Stan and its R package (RStan) (Stan Development Team, 2016), which could solve this problem. For popularization of the COM method, further case studies are needed to examine its applicability to several species in different regions.

\section{Acknowledgement}

This study was supported by JSPS KAKENHI Number 25850112, 25252029 and 15K07483, and the research project of the Agriculture, Forestry and Fisheries Research Council entitled as "Development of technology for impacts, mitigation and adaptation to climate change in the sectors of agriculture, forestry, and fisheries". 


\section{References}

Auten, J. T. (1945) Prediction of site index for yellow-poplar from soil and topography, J. For. 43: $662-668$.

Chen, J. and Abe, N. (1999) Site classification for sugi plantation using GIS, J. For. Plann. 5: 1-8.

Clutter, J.L., Fortson, J.C., Pienaar, L.V., Brister, G.H. and Bailey, R.L. (1983) Timber management: a quantitative approach, John Wiley \& Sons, New York.

Corona, P., Scotti, R. and Tarchiani, N. (1998) Relationship between environmental factors and site index in Douglas-fir plantations in central Italy, For. Ecol. Manage. 110: 195-207.

Curt, T., Bouchaud, M. and Agrech, G. (2001) Predicting site index of Douglas-fir plantations from ecological variables in the Massif Central area of France, For. Ecol. Manage. 149: 61-74.

Davis, L.S. and Johnson, K.N. (1987) Forest Management, McGraw-Hill, New York third edition.

Gelman, A., Carlin, J.B., Stern, H.S. and Rubin, D.B. (2003) Bayesian Data Analysis, Chapman \& Hall, Boca Raton, USA 2n̂d edition.

Green, R.N., Marshall, P.L. and Klinka, K. (1989) Estimating site index of Douglas-fir (Pseudotsuga menziessi [Mirb] Franco) from ecological variables in southwestern British Columbia, For. Sci. 35: $50-63$.

Hägglund, B. (1981) Evaluation of forest site productivity, For. Abst. 42: 515-527.

Hunter, I.R. and Gibson, A.R. (1984) Predicting Pinus radiata site index from environmental variables, New Zealand J. For. Science. 14: 53-64.

Kayahara, G.J., Klinka, K. and Marshall, P.L. (1998) Testing site index-site-factor relationship for predicting Pinus contorta and Picea engelmanii $\times$ P. glauca productivity in central British Columbia, For. Ecol. Manage. 110: 141-150.

Klinka, K. and Carter, R.E. (1990) Relationship between site index and synoptic environmental factors in immature coastal Douglas-fir stands, For. Sci. 36: 815-830.

Klinka, K. and Feller, M.C. (1984) Principles used in selecting tree species for regeneration of forest sites in southwestern British Columbia, For. Chron. 60: 77-85.

Mitsuda, Y. (2014) Development of a new method for modeling site index using the digital stereo aerial photo-derived spatial distribution of canopy height, FORMATH 13: 41-59.

Mitsuda, Y. and Ito, S. (2015) Modifying the site index model of sugi planted forests in Miyazaki Prefecture considering the effects of DEM quality and scale of digital terrain analysis, J. For. Plann. 20: 45-51.

Mitsuda, Y., Ito, S. and Iehara, T. (2013) Methodology for regional forest reallocation using criteria and indicators of the Montreal Process, Landsc. Ecol. Manage. 18: 123-137, (In Japanese with English summary).

Mitsuda, Y., Ito, S., Matsushita, K., Fukuzato, K., Taniguchi, Y., Mizoue, N. and Shimizu, O. (2003), ESC (ecological site classification) based DSS for Japanese forest landscape suffering frequent rainstorms, In CD Proceedings of a Transdisciplinary Conference on Decision Support for Multiple Purpose Forestry Vienna.

Mitsuda, Y., Ito, S. and Sakamoto, S. (2007) Predicting the site index of sugi plantations from GIS-derived environmental factors in Miyazaki Prefecture, J. For. Res. 12: 177-186.

Mitsuda, Y. and Kitahara, F. (2015) Preliminary analysis on site index of sugi (Cryptomeria japonica) planted forests using the National Forest Inventory data in Kyushu Island, FORMATH 14: 20-26. 
Mitsuda, Y., Yoshida, S. and Imada, M. (2001) Use of GIS-derived environmental factors in predicting site indices in Japanese larch plantations in Hokkaido, J. For. Res. 6:87-93.

Monserud, R.A., Moody, U. and Breuer, D.W. (1990) A soil-site study for inland Douglas-fir, Can. J. For. Res. 20: 686-695.

Nishizawa, M., Mashimo, Y. and Kawabata, K. (1965) Estimation method of site index by quantification, Bull. Gov. For. Exp. Stn. 176: 1-54.

Richards, F.J. (1959) A flexible growth function for empirical use, J. Exp. Bot. 10: 134-159.

Stan Development Team (2016) Stan: A C++ Library for Probability and Sampling, Version 2.11.

Takeshita, K., Fukushima, T., Hagiwara, Y. and Saijo, T. (1966) Stand analysis concerning estimation of forest-land productivity, Bull. Fukuoka-pref. For. Exp. Stn. 18: 41-76, (In Japanese with English summary).

Takeshita, K., Nakanjima, Y., Nagahama, M. and Higuchi, S. (1960) Edaphological studies on micro-topography and forest soil and their relationship to the growth of sugi Crypotmeria races (I), Bull.Fukuoka-pref.For.Exp.Stn. 12: 1-162, (In Japanese with English summary).

Trimble, Jr., G.R. and Weitzman, S. (1956) Site index of upland oaks in the northern Appalachians, For. Sci. 2: 162-173.

Van Oijen, M., Rougier, J. and Smith, R. (2005) Bayesian calibration of process-based forest models: bridging the gap between models and data, Tree Physiol. 25: 915-927.

Wang, G.G. (1995) White spruce site index in relation to soil, understory vegetation, and foliar nutrients, Can. J. For. Res. 25: 29-38.

Wang, G.G., Huang, S., Monserud, R.A. and Klos, R.J. (2004) Lodgepole pine site index in relation to synoptic measures of climate, soil moisture and soil nutrients, For. Chron. 80: 678-686. 